\title{
The Use of Left Ventricular Myocardial Stiffness Index as a Predictor of Myocardial Performance in Patients with Systemic Hypertension
}

\author{
Muataz F. Hussein', Asia H. Al-Mashhadani², Samar I. Essa' \\ ${ }^{1}$ Department of Medicine, College of Medicine, Baghdad University, Baghdad, Iraq \\ ${ }^{2}$ Department of Physics, College of Science, Baghdad University, Baghdad, Iraq \\ Email: assia19662006@yahoo.com
}

Received 28 June 2014; revised 20 July 2014; accepted 11 August 2014

Copyright (C) 2014 by authors and Scientific Research Publishing Inc.

This work is licensed under the Creative Commons Attribution International License (CC BY). http://creativecommons.org/licenses/by/4.0/

(c) (i) Open Access

\section{Abstract}

Our aim was to investigate the changes in the myocardium stiffness index for patients suffering from systemic hypertension, and to assess their left ventricular performance. We studied 263 hypertensive patients and 166 healthy subjects as a control group. By using conventional Doppler echocardiography, the following parameters were measured-Left ventricular end diastolic diameter, left ventricular end systolic diameter, transmitral early velocity, isovolumic relaxation time, and isovolumic contraction time. Tissue Doppler imaging (TDI) was used in the measurements of the early mitral annular velocity (Ea) and the diastolic stiffness was obtained by calculating the ratio $\mathrm{E} \backslash \mathrm{Ea} \backslash \mathrm{LVIDd}$. Index myocardial performance (IMP) was calculated according to following equation (IVCT + IVRT)/ET for both the control group and hypertensive group. Results reveal that the differences in the average value of transmitral early filling velocity (E) between patients and control groups for age range $(20-49)$ and $(50-80)$ were $(-1.91 \%)$ and $(-3.69 \%)$ respectively with $p$ value $>0.05$ for both groups, and the changes in LVIDd between patients and control groups were $0.42 \%$ and $1.29 \%$ for age ranges $(20-49)$ and $(50-80)$ respectively with $p$ value $>0.05$ for both age groups. A significant difference in IMP between patients and controls has also been observed and the changes were $29.54 \%$ and $30.43 \%$ for age range $(20-49)$ and $(50-80)$ respectively with $p$ value $<0.05$ for both groups. The mean values for $E / E a$ ratio and for the measured LV stiffness index were significantly higher in hypertensive patients compared with control group (p value $<0.05$ ) for both age groups. In conclusion, LV myocardial diastolic stiffness index and IMP are increased in hypertensive patients. 


\section{Keywords}

\section{Left Ventricular Stiffness, Systemic Hypertension}

\section{Introduction}

Hypertensive heart disease is defined as the response of the heart to afterload imposed on the left ventricle by the progressively increasing arterial pressure and total peripheral resistance produced by hypertensive vascular disease [1]. Uncontrolled and prolonged elevation of BP can lead to a variety of changes in the myocardial structure, coronary vasculature, and conduction system of the heart. These changes in turn can lead to the development of left ventricular hypertrophy (LVH), coronary artery disease (CAD), and various conduction system diseases [2]. Whether LV diastolic dysfunction is caused directly by raised blood pressure or by structural changes related to LV hypertrophy remains controversial but hypertensive patients may present with normal transmitral inflow filling patterns or with the more typical presentation of diastolic dysfunction [3].

Doppler echocardiography is the most important clinical tool available for the diagnosis of diastolic dysfunction. Echocardiography is widely available, portable, and biologically safe and is also capable of excluding other important causes of heart failure, such as valvular disease [4].

Doppler echocardiography has become the common noninvasive method involved in the measurement of the transmitral flow velocity, the isovolumic relaxation time (IVRT) and left atrial pressure [5] [6].

IMP was described as Doppler echocardiographic myocardial performance index combining time intervals related to systolic and diastolic function that reflects global cardiac function. The IMP is defined as the sum of isovolumetric contraction time (IVCT) with isovolumetric relaxation time (IVRT) divided by left ventricular ejection time (ET) [7] [8]. Tissue Doppler echocardiography (TDE) directly measures myocardial velocities. Diastolic tissue Doppler velocities reflect myocardial relaxation, and in combination with conventional Doppler measurements, the index of diastolic stiffness can be calculated. The measurements have included the calculation of the ratio of transmitral early diastolic velocity/mitral annular early diastolic velocity (E/Ea) which has been reported to give noninvasive estimation of left ventricular (LV) filling pressure [9]. The ratio E/Ea to LV end diastolic diameter (LVIDd), represents the index of diastolic stiffness ((E/Ea)/LVIDd) that represents a pressure- volume relationship of the left ventricle. This echo Doppler index of diastolic LV myocardial stiffness was previously studied in patient with left ventricular hypertrophy [10].

\section{Patients and Methods}

In the present study, patients with hypertension were chosen. Patients were examined for other diseases which can interfere with LV stiffness. A total of 429 subjects were included in the study, 263 patients with an established diagnosis of hypertension (HT group), (105 males and 158 females of mean age of $54.07 \pm 12.7$ ) and 166 healthy subjects (mean age $40.06 \pm 12.8$, 53 males and 113 females) as control group. The study was performed during the period from January 2013 until March 2014, at the echocardiography unit at Baghdad teaching hospital/Medical city/Baghdad.

The plan of the study consisted of the following steps:

Case history, Electrocardiography (ECG), Chest X-ray and Echocardiographic examination.

The echocardiography was performed for each subject using SONOACE X8 equipped with a transducer operating at 2 - $5 \mathrm{MHz}$. During quite respiration with the patients lying on the left decubitus position, left ventricular internal dimensions at diastole (LVIDd) and systole (LVIDs) were measured, using M-mode echocardiography [11]. Pulse wave Doppler tracing of the transmittal flow velocity was obtained from the apical fourchamber view measurement of the early mitral velocity $\mathrm{E}$ and the late velocity $\mathrm{A}$ at atrial contraction [12].

Mitral annulus velocity measurements were obtained from the apical four-chamber view by tissue Doppler image, using a $1-2 \mathrm{~mm}$ sample volume placed at the lateral side of the mitral valve annulus. The TDI mitral annular velocities were measured including the early annular velocity (Ea) [13]. All TDI velocities were taken as an average of the lateral readings of five cardiac cycles. The mean values of Ea velocities were used to calculate the $\mathrm{E} / \mathrm{Ea}$ ratio. Myocardial diastolic stiffness index was calculated using the equation of (Stiffness index = [(E/Ea)/LVIDd]) [14]. 
By using the apical view at aortic valve isovolumetric contraction time (IVCT), isovolumetric relaxation time (IVRT) and ejection time (ET) were measured from the interval between two mitral inflow periods (Figure 1), several investigators used IMP to assess left ventricle function, most of them showed that IMP gives a good assessment of left ventricular dysfunction and its severity. IMP can be calculated by using the equation that IMP = (IVCT + IVRT)/ET [15].

All values were expressed as mean values with standard deviations. The comparison between the mean values for both groups was tested by paired student's t-test. $p$ value $<0.05$ was considered as the level of significance.

\section{Results}

The study included 263 hypertensive patients (158 females and 105 males, age $54.07 \pm 12.7$ years) and 166 healthy subjects (113 females and 53 males, age $40.06 \pm 12.8$ years).

Table 1 shows conventional echo-Doppler findings obtained from the study population. The change in transmitral early velocity (E) between HT patients and control group is small $(-1.91 \%)$ and it is insignificant, p value $>0.05$. The late (active) filling velocity (A) is higher in the patient group by (29.44\%) with strongly significant $p$ value $<0.05$. The change in LVIDd between patients and controls is $0.42 \%$ with insignificant $p$ value $>0.05$, while the difference in LVIDs is $4.69 \%$ with significant $\mathrm{p}$ value $<0.05$, for the age range 20 - 49 years.

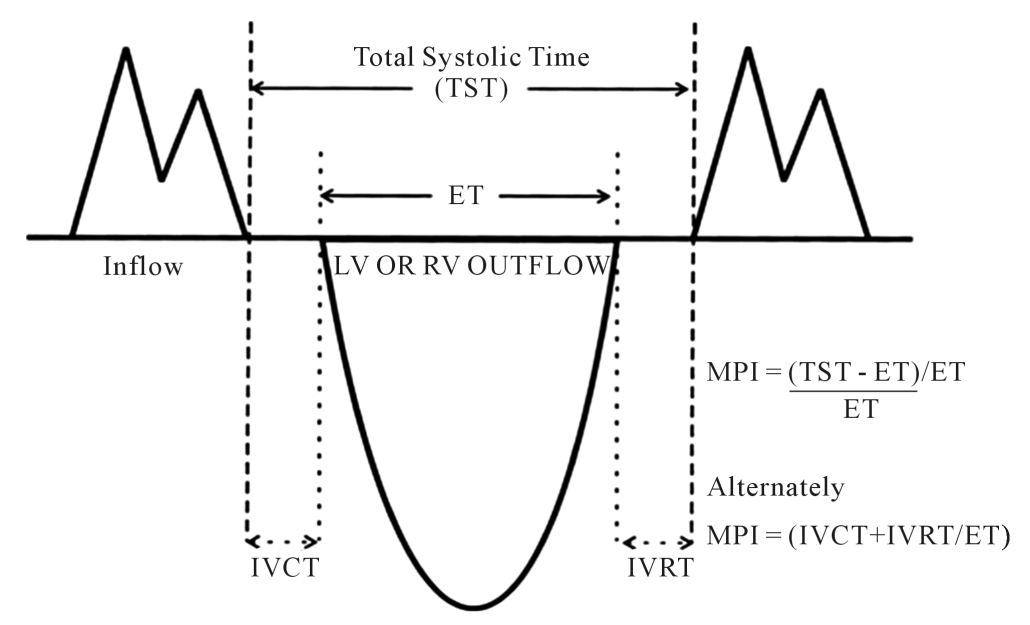

Figure 1. Measurement of Doppler interval from aortic valve (AV) opening to aortic valve (AV) closure.

Table 1. Conventional M mode and two dimensional echocardiographic findings.

\begin{tabular}{|c|c|c|c|c|}
\hline Variable & Control Mean \pm SD & HT Mean \pm SD & Change $\%=(\mathrm{HT}-\mathrm{C}) / \mathrm{C} \times 100$ & p Value \\
\hline \multicolumn{5}{|c|}{ The age range from 20 to 49 years } \\
\hline $\mathrm{E}(\mathrm{mm} / \mathrm{s})$ & $73.82 \pm 8.74$ & $72.41 \pm 16.26$ & $-1.91 \%$ & $>0.05$ \\
\hline $\mathrm{A}(\mathrm{mm} / \mathrm{s})$ & $55.027 \pm 9.29$ & $71.22 \pm 14.75$ & $29.44 \%$ & $<0.05$ \\
\hline LVIDd (mm) & $4.76 \pm 0.59$ & $4.780 \pm 0.63$ & $0.42 \%$ & $>0.05$ \\
\hline LVIDs (mm) & $2.98 \pm 0.52$ & $3.12 \pm 0.59$ & $4.69 \%$ & $<0.05$ \\
\hline \multicolumn{5}{|c|}{ The age range from 50 to 80 years } \\
\hline $\mathrm{E}(\mathrm{mm} / \mathrm{s})$ & $70.704 \pm 12.151$ & $68.098 \pm 16.34$ & $-3.69 \%$ & $>0.05$ \\
\hline $\mathrm{A}(\mathrm{mm} / \mathrm{s})$ & $62.34 \pm 13.25$ & $79.42 \pm 14.95$ & $27.39 \%$ & $<0.05$ \\
\hline LVIDd (mm) & $4.64 \pm 0.45$ & $4.706 \pm 0.69$ & $1.29 \%$ & $>0.05$ \\
\hline LVIDs (mm) & $3.075 \pm 0.491$ & $3.082 \pm 0.64$ & $0.32 \%$ & $>0.05$ \\
\hline
\end{tabular}

$\mathrm{p}<0.05$ = significant; $\mathrm{p}>0.05$ = insignificant. 
For age range 50 - 80, the change in transmitral early velocity (E) between HT patients and control group was $-3.69 \%$, also insignificant with $p$ value $>0.05$. In contrast to the early filling (E), the late (active) filling velocity (A) is higher in the patient group by $27.39 \%$ with strongly significant p value $<0.05$, while LVIDd and LVIDs showed insignificant difference between both groups, $\mathrm{p}$ value $>0.05$.

For age range 20 - 49, results in Table 2 reveal the difference in IVCT between patients and controls were $12.36 \%$ giving significant $\mathrm{p}$ value $<0.001$, while change percent in IVRT and ET are $18.48 \%$ and $-10.59 \%$ respectively and strongly significant p value $<0.001$ for both, consequently the difference in IMP value is $29.54 \%$ and it was also strongly significant $\mathrm{p}$ value $<0.001$.

The difference in IVCT between patients and controls was $0.65 \%$ giving insignificant $\mathrm{p}$ value $>0.05$ in the age range 50 - 80 years, while change percents in IVRT and ET are $13.87 \%$ and $-15.06 \%$ respectively and both of them are strongly significant $p$ value $<0.001$, consequently IMP value is $30.43 \%$ and it was also strongly significant p value $<0.001$ (Table 2).

\section{IMP with Stiffness Index}

The change in IMP with stiffness index was shown in Figure 2. For age range 20 - 49, the graph shows that almost no change in the slope between control and patients with a jump between them as the curve for patients starts at higher values of IMP.

Figure 3 shows the change in IMP with stiffness index for age range (50 - 80) with a significant increase in the slope for control and a slight decrease in the slope of patients group.

For age range (20 - 49), mitral annular early diastolic velocity Ea was significantly lower in the hypertension

Table 2. Doppler echocardiography showing change \% for (IVCT, IVRT, ET and IMP).

\begin{tabular}{cccc}
\hline Variable & Control Mean \pm SD & HT Mean \pm SD & Change $\%=($ HT-C) $/$ C $\times 100$ \\
\hline & & The age range from 20 to 49 years & \\
\hline IVRT & $76.133 \pm 16.83$ & $90.206 \pm 20.061$ & $18.48 \%$ \\
IVCT & $53.2 \pm 11.76$ & $59.782 \pm 18.46$ & $12.36 \%$ \\
ET & $298.191 \pm 49.149$ & $266.59 \pm 41.292$ & $<.05 \%$ \\
IMP & $0.445 \pm 0.1088$ & $0.576 \pm 0.143$ & $29.54 \%$ \\
\hline IVRT & $82.84 \pm 18.168$ & The age range from 50 to 80 years & $13.87 \%$ \\
IVCT & $59.72 \pm 13.84$ & $94.33 \pm 20.98$ & $<0.05$ \\
ET & $309.659 \pm 45.929$ & $60.112 \pm 17.48$ & $-15.05 \%$ \\
IMP & $0.466 \pm 0.099$ & $262.99 \pm 39.074$ & $<0.05$ \\
\hline
\end{tabular}

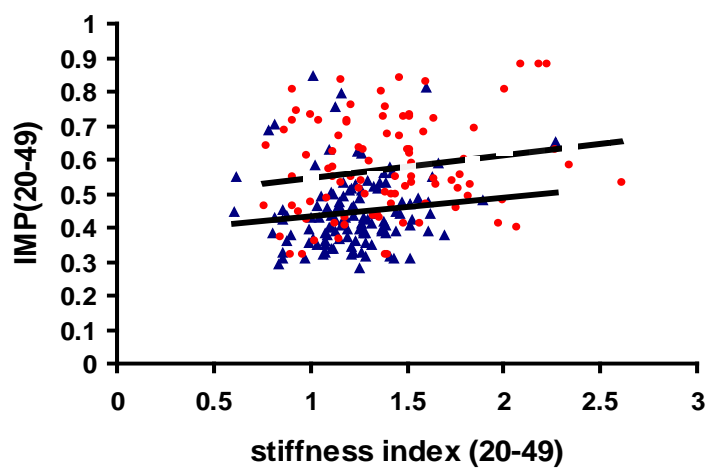

$y(c)=0.0561 x+0.3773$

$y(p)=0.0668 x+0.4826$

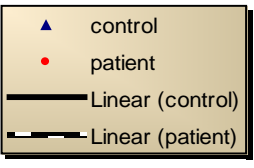

Figure 2. Relationship between stiffness index and IMP in controls and patients groups. Age between 20 - 49 years. 


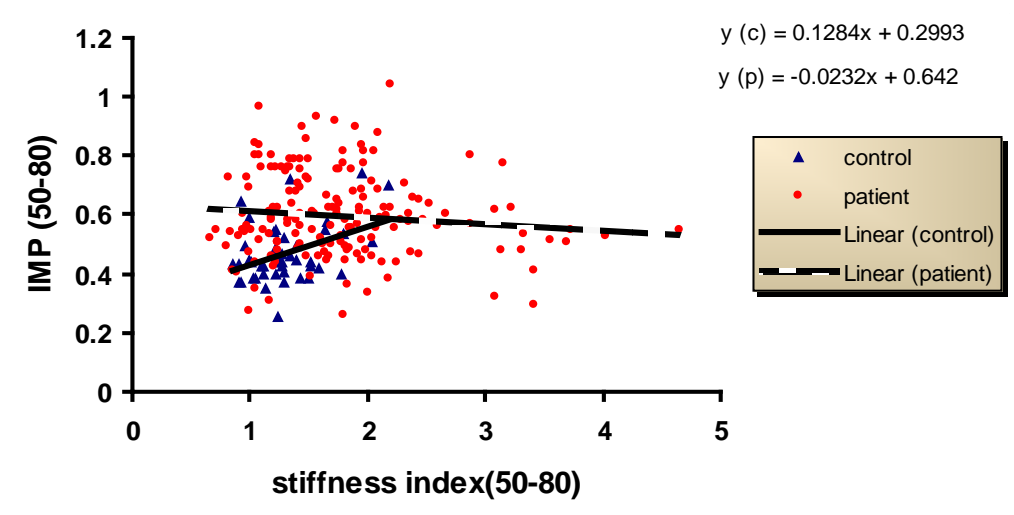

Figure 3. Relationship between stiffness index and IMP in controls and patients groups. Age are between 50 - 80 years.

group compared with the control group by $-14.09 \%$, $p$ value $<0.05$. The LV stiffness index and E/Ea were significantly higher in hypertensive compared with control by $40 \%$ and $15.55 \%$ respectively, $\mathrm{p}<0.001$.

But for age range 50 - 80, mitral annular early diastolic velocity Ea was significantly lower in the hypertensive group compared with the control group by $-25.166 \%$, p value $<0.05$. The LV stiffness index and E/Ea were significantly higher in hypertensive compared with control by $35.38 \%$ and $34.61 \%$ respectively, p value < 0.05) (Table 3).

\section{Discussion}

In this study, we have investigated the effect of hypertension on the cardiac muscle by assessing the myocardial stiffness through the use of tissue Doppler by the measurement of the change in the annular velocity in relation with the myocardium performance and the mechanical movement together with the geometrical changes. It is well known that a change in the myocardial stiffness, geometry and performance caused by overload can be related to fibrosis and fibrosis can be generated by increased collagen content in the muscle, which can increase stiffness [16].

Stiffness affects the myocardial movement and the change in the movement can be detected and measured by tissue Doppler [17].

The annular tissue Doppler shows the change in the movement speed throughout the cardiac cycle. A decrease in Ea was observed in HT patients and in the elderly (Table 3).

We have divided the study population according to their age, this has the benefit of identifying the stiffness origin recognizing whether it is originated from the disease or influenced by aging in addition to finding the effect of aging on the disease. This can be well seen on the reduction in E wave which is not high in patients compared with controls and for both age groups which did not exceed $-1.91 \%,-3.69 \%$ for young and old group respectively (because our patients had short HT duration), but a higher change is more clear in A wave $29.44 \%$ and $27.39 \%$ for the young and old age groups respectively (Table 1). The higher A wave may be caused by less blood volume transferred to the LV during the $\mathrm{E}$ wave which may be caused by the decrease in suction during early diastole, the left atrial to left ventricular (LV-LA) pressure gradient at the time of mitral valve opening is also decreased (i.e. deceleration time is prolonged, unless left ventriclar stiffness is significantly increased) [18], and to the fact that most of the transmitral blood filling for the LV is attributed to $\mathrm{E}$ wave rather than $\mathrm{A}$ wave , so in order to compensate the lack of blood to the $\mathrm{LV}$ the late filling velocity A wave must increase more than what $\mathrm{E}$ wave decreases. So A wave should be of high speed enough to compensate the lack in the early transmitral blood reduction. This result is in agreement with previous study by Masanori et al. (2006) [19], these results are shown in Table 1.

In most cases of HT, we may find an impairment in the LV function before LA impairment appeared (in the absence of other cardiac diseases) and this is due to the pressure overload which influences the LV more than LA probably causing reduced E/A which indicate the diastolic dysfunction reflected on diastolic performance caused by reduced E and increased A. This result is in agreement with previous studies by Garcia et al. 1998 [20]. 
Table 3. Tissue Doppler echocardiography showing change \% for (Ea, and E/Ea).

\begin{tabular}{|c|c|c|c|c|}
\hline Variable & Control Mean \pm SD & HT Mean \pm SD & Change (HT-C) $/ \mathrm{C} \times 100$ & $\mathrm{p}$ Value \\
\hline \multicolumn{5}{|c|}{ The age range from 20 to 49 years } \\
\hline $\mathrm{Ea}(\mathrm{mm} / \mathrm{s})$ & $13.208 \pm 2.47$ & $11.34 \pm 2.7$ & $-14.09 \%$ & $<0.05$ \\
\hline $\mathrm{E} / \mathrm{Ea}$ & $5.72 \pm 0.99$ & $6.61 \pm 1.62$ & $15.55 \%$ & $<0.05$ \\
\hline Stiffness index & $1.21 \pm 0.246$ & $1.404 \pm 0.38$ & $40 \%$ & $<0.05$ \\
\hline \multicolumn{5}{|c|}{ The age range from 50 to 80 years } \\
\hline $\mathrm{Ea}(\mathrm{mm} / \mathrm{s})$ & $12.045 \pm 1.94$ & $9.017 \pm 2.57$ & $-25.166 \%$ & $<0.05$ \\
\hline $\mathrm{E} / \mathrm{Ea}$ & $5.98 \pm 1.23$ & $8.059 \pm 2.71$ & $34.61 \%$ & $<0.05$ \\
\hline Stiffness index & $1.303 \pm 0.313$ & $1.763 \pm 0.69$ & $35.38 \%$ & $<0.05$ \\
\hline
\end{tabular}

The reduction in $\mathrm{E}$ has an effect on the increase in A wave because more LV filling volume is due to the passive E velocity, so for A wave to compensate it should be of high speed enough to compensate the lack in the early trans-mitral blood reduction.

Due to the increase in the LV overload the muscle passes through several stages before the heart failure ensue. Changes such as hypertrophy occur as a response of overload and if the over load persists for long time the myocardium will gradually loose ability of contraction which eventually leading to heart failure. During these changes fibrosis might be increased with time caused by the formation of collagen in the myocardium and eventually leads to the muscle stiffness [21].

It is rather feasible to get higher increase in the percentage of stiffness index for HT patient over control for the young age (40\%) (Table 3) this is because in the older age group the myocardium within certain extent is stiffened already with age without the pressure overload so the increase in the stiffness caused by hypertension over the age-related stiffness will possibly be less than what we can observe for the younger group, as the myocardium of the latter is still intact and flexible so an increase in the overload will appear more significant [22] (Table 3).

Because our patients hypertension was not chronic (the HT duration is less than 5 years) changes in the chamber size have not been occurred yet; for this reason the difference in LVIDd between patients and controls is very small and insignificant (Table 1). This is applicable for both age groups. The same result was also observed for LVIDs for old age group as there is insignificant difference between patients and control in contrast with the younger age group which gives significant difference between patient and control (Table 2). These results indicate that patients with HT have developed a slight change in the chamber size in the younger age group and insignificant change in the old age group. This is because that our patients have short HT duration and the effect has appeared on the LVIDs for the younger group age 20 - 49 because their myocardium is still with high flexibility so a mild increase in the stiffness can influence the muscle contraction.

Because the change in the LVIDd chamber size is very small and insignificant, $0.42 \%, 1.29 \%$ for both groups young and old age groups respectively, then the change in the stiffness index is attributed to the change in E/Ea which represents the change in the tansmitral pressure (dp) rather than the change in LVIDd, and because the change in $\mathrm{E}$ is also small $-1.91 \%$ and $-3.69 \%$ for both groups young and old age groups respectively (Table 1 ) then the change in the stiffness index is mainly due to the change in Ea as it has more changes than the two previous variables, $-14.09 \%$ and $-25.166 \%$ for young and old age group respectively than both LVIDd and filling velocity E.

The advantage of TDE in defining the value of IMP is its ability to simultaneously record systolic and diastolic flows from the systolic-diastolic displacements of ventricular wall recorded at the level of the lateral mitral annulus [23]. This site appears to be more reliable than others, according to Harada and coworkers [24].

A rapidly determined index of ventricular function has been derived by comparing the total systolic time from mitral valve closure to mitral valve opening with the systolic time involved in actual aortic flow (ejection time).

Left ventricular relaxation time IVRT and contraction time IVCT are prolonged because of the increased proportion of collagen fibers and the slow sequence of electrical repolarization that leads to non-uniform relaxation, which is in agreement with the previous study by Mario 2006 [25]. 
As IMP is defined as the sum of IVCT and IVRT divided by ejection time ET, so slowing in the sequence of the electrical re-polarization in the stiff myocardium can increase the IVCT and IVRT. IVRT measurement provides into the rate of early diastolic left ventricle relaxation. When the relaxation is prolonged, mitral valve opening is delayed and IVRT is increased. Isovolumic relaxation time is an indicator of the rate of myocardial relaxation. A major limitation is the fact that multiple factors influence the duration of the IVRT. Impaired relaxation lengthens IVRT, and IVRT increase with age and is sensitive to changes in both heart rate and systolic function [26].

As a result, the change in IMP follows the increase in IVRT and IVCT with reduced ET, thus IMP will increase with the increase in the stiffness index as shown in the (Table 2). The increase in IMP is an indicator for cardiac dys function, these results are in agreement with the previous study of Nixon et al. 1994 [27].

In Figure 2, the increase in the slope in the graph of patients and controls for the young age group indicates a progressive deterioration in the cardiac muscle performance with the increase in the stiffness index. In the old age 50 - 80, the graph of control was rising steeply with the increase in the stiffness index this can give the indication of fast deterioration in the old age controls when develop stiffness. As for controls of old age patients the line of the graph is slightly descending or almost zero slope which is probably caused by the beginning of pseudonormalization [28].

\section{Conclusions}

The diastolic stiffness was significantly higher in hypertensive patients compared to healthy subjects. This may be due to the fact that patients with hypertension experienced certain morphologic and structural changes of the left ventricle including left ventricular hypertrophy reducing LV compliance and increasing myocardial stiffness.

From the results, it can be concluded that IMP appears to be a good measure of global left ventricular function (systolic and diastolic function), and is a sensitive and accurate evaluation of disease states depending on age.

\section{References}

[1] Izzo, J.L. and Gradman, A.H. (2004) Mechanisms and Management of Hypertensive Heart Disease: From Left Ventricular Hypertrophy to Heart Failure. Medical Clinics of North America, 88, 1257-1271.

[2] Guyton, A.C. and Hall, J.E. (2000) Text Book of Medical Physiology. 10th Edition, Philadelphia B-Sounders Company, 96-109, 229-232.

[3] Devereux, R.B. and Roman, M.J. (1999) Left Ventricular Hypertrophy in Hypertension: Stimuli, Patterns, and Consequences. Hypertension Research, 22, 1-9. http://dx.doi.org/10.1291/hypres.22.1

[4] Cohen, G.I., Pietrolungo, J.F., Thomas, J.D. and Klein A.L. (1996) A Practical Guide to Assessment of Ventricular Diastolic Function Using Doppler Echocardiography. Journal of the American College of Cardiology, 27, 1753-1760. http://dx.doi.org/10.1016/0735-1097(96)00088-5

[5] Nishimura, R.A., Abel, M.D., Hatle, L.K. and Tajik, A.J. (1989) Assessment of Diastolic Function of the Heart: Background and Current Applications of Doppler Echocardiography. Part II. Clinical Studies. Mayo Clinic Proceedings, 64, 181-204. http://dx.doi.org/10.1016/S0025-6196(12)65673-0

[6] Stoddard, M.F., Pearson, A.C., Kern, M.J., Ratcliff, J., Mrosek, G. and Labovitz, A.J. (1989) Influence of Alteration in Preload on the Pattern of Left Ventricular Diastolic Filling Assessed by Doppler Echocardiography in Humans. Circulation, 79, 1226-1236. http://dx.doi.org/10.1161/01.CIR.79.6.1226

[7] Tei, C. (1995) New Non-Invasive Index for Combined Systolic and Diastolic Ventricular Function. Journal of Cardiology, 26, 135-136.

[8] Krumholz, H.M., Larson, M. and Levy, D. (1995) Prognosis of Left Ventricular Geometric Patterns in the Framingham Heart Study. Journal of the American College of Cardiology, 25, 879-884. http://dx.doi.org/10.1016/0735-1097(94)00473-4

[9] Ommen, S.R., Nishimura, R.A. and Appleton, C.P. (2000) Clinical Utility of Doppler Echocardiography and Tissue Doppler Imaging in the Estimation of Left Ventricular Filling Pressures: A Comparative Simultaneous Doppler-Catheterization Study. Circulation, 102, 1788-1794. http://dx.doi.org/10.1161/01.CIR.102.15.1788

[10] Westermann, D., Kasner, M. and Steendijk, P. (2008) Role of Left Ventricular Stiffness in Heart Failure with Normal Ejection Fraction. Circulation, 117, 2051-2060. http://dx.doi.org/10.1161/CIRCULATIONAHA.107.716886

[11] Maron, B.J., Gottdiener, J.S. and Epstein, S.E. (1981) Patterns and Significance of Distribution of Left Ventricular Hypertrophy in Hypertrophic Cardiomyopathy. A Wide Angle, Two-Dimensional Echocardiographic Study of 125 Pa- 
tients. American Journal of Cardiology, 48, 418-428. http://dx.doi.org/10.1016/0002-9149(81)90068-0

[12] Mulvagh, S., Quinones, M.A. and Kleiman, N.S. (1992) Estimation of Left Ventricular End-Diastolic Pressure from Doppler Transmitral Flow Velocity in Cardiac Patients Independent of Systolic Performance. Journal of the American College of Cardiology, 20,112-119. http://dx.doi.org/10.1016/0735-1097(92)90146-E

[13] Srivastava, P.M., Burrell, L.M. and Calafiore, P. (2005) Lateral vs Medial Mitral Annular Tissue Doppler in the Echocardiographic Assessment of Diastolic Function and Filling Pressures: Which Should We Use? European Journal of Echocardiography, 6, 97-106. http://dx.doi.org/10.1016/j.euje.2004.07.005

[14] King, G.J., Murphy, R.T. and Almuntaser, I. (2008) Alterations in Myocardial Stiffness in Elite Athletes Assessed by a New Doppler Index. Heart, 94, 1323-1325. http://dx.doi.org/10.1136/hrt.2008.142083

[15] Yilmaz, R., Seydaliyeva, T., Unlu, D. and Ulucay, A. (2004) The Effect of Left Ventricular Geometry on Myocardial Performance Index in Hypertensive Patients. Anadolu Kardiyoloji Dergisi, 4, 217-227.

[16] Kannel, W.B., Dawber, T.R., Kogan, A., Revotskie, N. and Stokes, J. (1961) Factors of Risk in the Development of Coronary Heart Disease. Six Years of Follow-Up Experience. Annals of Internal Medicine, 55, 33-50. http://dx.doi.org/10.7326/0003-4819-55-1-33

[17] Folkow, B. (1978) Cardiovascular Structural Adaptation: Its Role in the Initiation and Maintenance of Primary Hypertension. Clinical Science Molecular Medicine, 55, 3-22.

[18] Inouye, I., Massie, B. and Loge, D. (1984) Abnormal Left Ventricular Filling: An Early Finding in Mild to Moderate Systemic Hypertension. American Journal of Cardiology, 53, 120-126. http://dx.doi.org/10.1016/0002-9149(84)90695-7

[19] Nakamura, M., Wada, S. and Yamaguchi, T. (2006) Influence of the Opening Mode of the Mitral Valve Orifice on Intraventricular Hemodynamics. Annals of Biomedical Engineering, 34, 927-935.

[20] Garcia, M.J., Thomas, J.D. and Klein, A.L. (1998) New Doppler Echocardiographic Applications for the Study of Diastolic Function. Journal of the American College of Cardiology, 32, 865-875. http://dx.doi.org/10.1016/S0735-1097(98)00345-3

[21] Lips, D.J., de Windt, L.J., van Kraaij, D.J.W. and Doevedans, P.A. (2003) Molecular Determinants of Myocardial Hypertrophy and Failure: Alternative Pathways for Beneficial and Maladaptive Hypertrophy. European Heart Journal, 24, 883-896. http://dx.doi.org/10.1016/S0195-668X(02)00829-1

[22] Villari, B., Vassalli, G. and Schneider, J. (1997) Age Dependency of Left Ventricular Diastolic Function in Pressure Overload Hypertrophy. Journal of the American College of Cardiology, 29,181-186. http://dx.doi.org/10.1016/S0735-1097(96)00440-8

[23] Yamazaki, N., Mine, Y. and Sano, A. (1994) Analysis of Ventricular Wall Motion Using Color-Coded Using Tissue Doppler Imaging System. Japanese Journal of Applied Physics, 33, 3141-3146.

[24] Harada, K., Tamura, M. and Toyono, M. (2002) Comparison of the Right Ventricular Tei Index y Tissue Doppler Imaging to That Obtained by Pulsed Doppler in Children without Heart Disease. American Journal of Cardiology, 90, 566-569. http://dx.doi.org/10.1016/S0002-9149(02)02541-9

[25] Mario, J.G. (2006) Comprehensive Echocardiographic Assessment of Diastolic Function. Heart Failure Clinics, 2, 163-178. http://dx.doi.org/10.1016/j.hfc.2006.01.005

[26] Seok, M.K., Jong, W.H., Se-Joong, R. and Namsik, C. (1998) Index of Myocardial Performance Using Doppler-Derived Parameter in the Evaluation of Left Ventricular Function in Patient with Essential Hypertension. Yonsei Medical Journal, 39, 446-452.

[27] Nixon, J.V. and Burns, C.A. (1994) Cardiac Effects of Aging and Diastolic Dysfunction in the Elderly. In: Gaasch W.H. and Le Winter M., Eds., Heart Failure and Left Ventricular Diastolic Dysfunction, Lea and Febiger, Philadelphia, 427-435.

[28] Nearchou, N., Tsakiris, A. and Tsitsirikos, M. (2005) Tei Index as a Method of Evaluating Left Ventricular Diastolic Dysfunction in Acute Myocardial Infarction. The Hellenic Journal of Cardiology, 46, 35-42. 


\section{Abbreviations and Acronyms}

Ea: Peak mitral annular early diastolic velocity

E/Ea: The ratio of early diastolic mitral inflow velocity to early diastolic tissue Doppler mitral annular velocity TDI: Tissue Doppler Imaging

E: Peak mitral early filling velocity

A: Peak mitral atrial phase filling velocity

LV: Left Ventricular

LVIDd: Left Ventricular End Diastolic Diameter

LVIDs: Left Ventricular End Systolic Diameter

LVH: Left Ventricular Hypertrophy

IMP: Index myocardial Performance

IVCT: Isovolumetric Contraction time

IVRT: Isovolumetric Relaxation time

ET: Ejection Time

HT: Hypertension

BP: Blood Pressure 
Scientific Research Publishing (SCIRP) is one of the largest Open Access journal publishers. It is currently publishing more than 200 open access, online, peer-reviewed journals covering a wide range of academic disciplines. SCIRP serves the worldwide academic communities and contributes to the progress and application of science with its publication.

Other selected journals from SCIRP are listed as below. Submit your manuscript to us via either submit@scirp.org or Online Submission Portal.
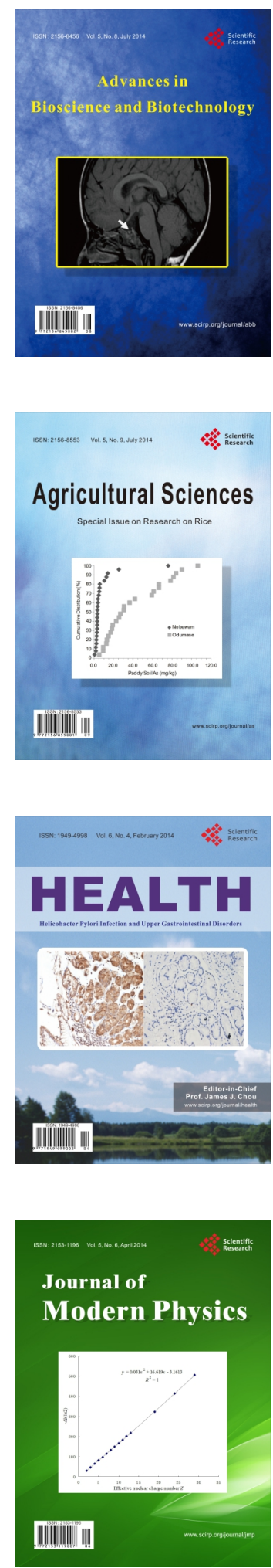
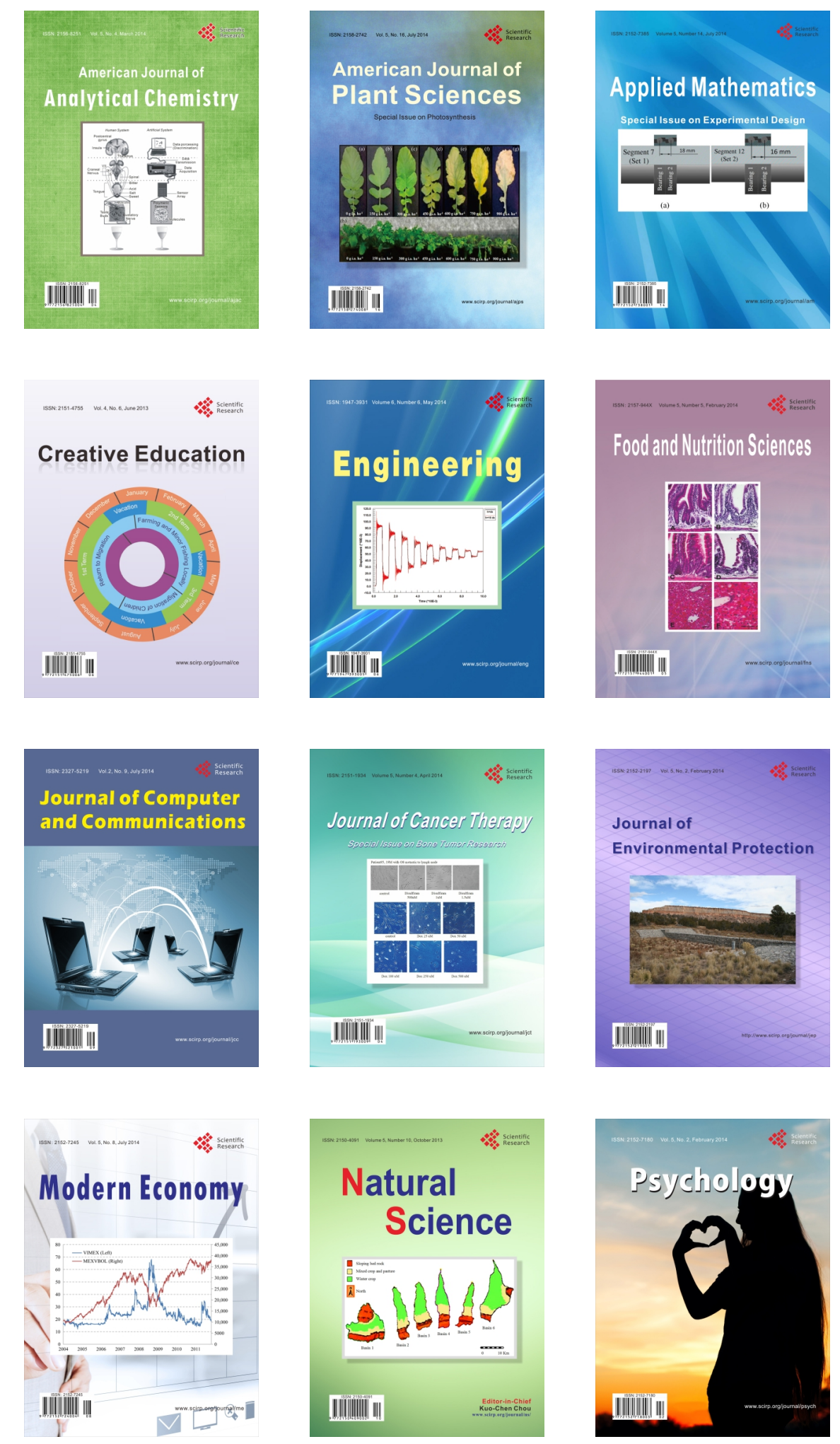